\title{
Geometric Phase for Analytically Solvable Driven Time-Dependent Two-Level Quantum Systems
}

\author{
I. Mendaš*, N. Burić, D.B. Popović, S. Prvanović and M. Radonjić \\ Institute of Physics, University of Belgrade, Pregrevica 118, 11080 Belgrade, Serbia \\ (Received May 11, 2013; revised version April 16, 2014; in final form May 23, 2014)
}

\begin{abstract}
Geometric phase for novel analytical solutions (Barnes and Das Sarma) of time-dependent two-level quantum systems is discussed, specifically for a general single-axis driving term, which is represented by a function $J(t)$ in the Hamiltonian, and its corresponding evolution operator. It is demonstrated how general results for corresponding phases (total, dynamic and geometric) can be obtained. Using a specific case, it was found that over time in which the driving field is appreciably different from zero, the corresponding geometric phase changes (in the specific example by $\Delta \beta \approx 0.8$ radians) thus enabling detection. The results are relevant to qubit control and to quantum computing applications.
\end{abstract}

DOI: 10.12693 /APhysPolA.126.670

PACS: 03.65.Aa, 03.65.Vf, 07.05.Dz

\section{Introduction}

Driven two-level systems are ubiquitous in quantum mechanics. Examples of exactly soluble two-level problems include Landau-Zener [1, 2], and Rabi [3] problems, Jaynes-Cummings [4] model, and others [6-11]. A new theoretical approach to the driven two-state system was recently introduced by Barnes and Das Sarma [12]. This uses a single-axis control field along the $z$ axis, which is represented by a driving field $J(t)$ in the Hamiltonian (the time varying energy splitting between states):

$$
H(t)=\frac{1}{2}\left[\begin{array}{cc}
J(t) & h \\
h & -J(t)
\end{array}\right] .
$$

Here $h$ represents a constant (an energy splitting between the two levels). The evolution operator, from an initial time $t=0$, to a later $t$, and corresponding to $H$ is represented by the $2 \times 2$ unitary matrix

$$
U(t)=\left[\begin{array}{cc}
U_{11} & -U_{21}^{*} \\
U_{21} & U_{11}^{*}
\end{array}\right],
$$

with $\left|U_{11}(t)\right|^{2}+\left|U_{21}(t)\right|^{2}=1$.

One then uses an innovative approach [12] to solve the corresponding time-dependent Schrödinger equation for the evolution operator and obtains the forms for the matrix elements of the evolution operator, Eqs. (12)-(16) in $[12]$.

It turns out that the driving field, $J(t)$, appearing in the Hamiltonian (1) is related to a function $q(t)$, with corresponding initial conditions given by Eq. (17) in [12], via

$$
J(t)=\frac{\ddot{q}+h^{2} q}{\sqrt{h^{2}\left(1-q^{2}\right)-\dot{q}^{2}}} .
$$

Such a simple prescription enables one to generate a number of novel, analytically solvable two-state problems together with their explicit solutions.

* corresponding author; e-mail: mendas@ipb.ac.rs
With such solutions one is able (at least in principle) to determine corresponding total, dynamic and geometric phases [13-19], in order to study and monitor the time development of the state vectors evolving under the Hamiltonian given by Eq. (1). This is of interest in the context of qubit control and quantum computing. The phases, for a pure state, are as follows. The total phase, $\varphi(0, t)$, of the state vector $|\Psi, t\rangle=U(t)|\Psi, 0\rangle$, accumulated during the evolution from an initial time 0 to a final $t$, is determined by the argument of the inner product

$$
\begin{aligned}
& \langle\Psi, 0 \mid \Psi, t\rangle \equiv r(0, t) \exp (\mathrm{i} \varphi(0, t)), \\
& \varphi(0, t)=\arg [\langle\Psi, 0 \mid \Psi, t\rangle] .
\end{aligned}
$$

Here we restrict ourselves to $r(0, t)>0$, so that the two states in question are not orthogonal, and the relative phases can be determined. By convention the result of the argument of the complex number $z, \arg (z)$, is always between $-\pi$ and $+\pi$. Thus the total phase is in radians and ranges across this interval. A part of the total phase is the dynamic phase, which is given by the time integral of the expectation value of the Hamiltonian (we use units $\hbar=1)$

$$
\delta(0, t) \equiv-\int_{0}^{t}\langle\Psi, \tau|H(\tau)| \Psi, \tau\rangle \mathrm{d} \tau .
$$

The geometric phase is then simply the surplus in the total phase over $\delta(0, t)$ :

$$
\beta(0, t) \equiv \varphi(0, t)-\delta(0, t) .
$$

Thus, in order to determine the geometric phase, the road is in principle simple. If one can solve the time-dependent Schrödinger equation (and this is precisely the case treated in [12]), the total phase is determined by the argument of the inner product $\langle\Psi, 0 \mid \Psi, t\rangle$, Eq. (4). Subsequently, the corresponding dynamic phase follows from the time integral of the expectation value of the Hamiltonian, Eq. (5). Finally, the difference between the two, Eq. (6), yields the geometric phase. There are numerous papers related to the geometric phase e.g. [20-22].

Here, in Sect. 2, we first present in some detail the theory on which the results of the calculations reported here 
are based, while in the subsequent Sect. 3 we describe the calculation of the phases. Finally a brief summary is presented.

\section{Spin polarization vector}

It is convenient to parametrize the time dependent Hamiltonian operator of a two-level system as

$$
H(t)=H_{0}(t) 1_{2}+\boldsymbol{H}(t) \cdot \boldsymbol{\sigma},
$$

where $\boldsymbol{\sigma}$ denotes the vector of three Pauli $2 \times 2$ matrices $\left(\sigma_{1}, \sigma_{2}, \sigma_{3}\right)$, and $1_{2}$ denotes the $2 \times 2$ unit matrix. In the case of the Hamiltonian (1) one has $H_{0}(t)=0$, $H_{1}(t)=\frac{1}{2} h, H_{2}(t)=0$ and $H_{3}(t)=\frac{1}{2} J(t)$.

Similarly one parametrizes the $2 \times 2$ unitary matrix Eq. (2) as

$$
U(t)=U_{0}(t) 1_{2}+\mathrm{i} \boldsymbol{U}(t) \cdot \boldsymbol{\sigma},
$$

with additional condition which stems from the unitarity condition of the evolution operator $\boldsymbol{U}(t): \boldsymbol{U}(t) \cdot \boldsymbol{U}(t)+$ $U_{0}^{2}=1$. In the case of (2) one has $U_{0}(t)=\Re\left\{U_{11}(t)\right\}$, $U_{1}(t)=\Im\left\{U_{21}(t)\right\}, U_{2}(t)=-\Re\left\{U_{21}(t)\right\}$, and $U_{3}(t)=$ $\Im\left\{U_{11}(t)\right\}$, where $\Re$ and $\Im$ denote the corresponding real and imaginary parts, respectively. Finally, the corresponding $2 \times 2$ density matrix is of the form

$$
\rho(t)=|\Psi, t\rangle\langle\Psi, t|=\frac{1}{2}\left(1_{2}+\boldsymbol{P}(t) \cdot \boldsymbol{\sigma}\right) .
$$

Here $\boldsymbol{P}(t)$ denotes the time dependent average spin polarization vector. Using this notation one has, from Eqs. (5) and (6)

$$
\begin{aligned}
& \varphi(0, t)=\arg (\operatorname{Tr}(U(t) \rho(0)))= \\
& \arctan \left(\frac{\boldsymbol{U}(t) \cdot \boldsymbol{P}(0)}{U_{0}(t)}\right) \equiv \arctan \alpha,
\end{aligned}
$$

with $\alpha=\alpha(t) \equiv\left(\Im\left\{U_{21}\right\}(t) P_{1}(0)-\Re\left\{U_{21}(t)\right\} P_{2}(0)+\right.$ $\left.\Im\left\{U_{11}(t)\right\} P_{3}(0)\right) / \Re\left\{U_{11}(t)\right\}$, and

$$
\begin{aligned}
& \delta(0, t)=-\int_{0}^{t} \operatorname{Tr}(H(\tau) \rho(\tau)) \mathrm{d} \tau \\
& =-\int_{0}^{t}\left(H_{0}(\tau)+\boldsymbol{H}(\tau) \cdot \boldsymbol{P}(\tau)\right) \mathrm{d} \tau \\
& =-\frac{1}{2} h \int_{0}^{t} P_{1}(\tau) \mathrm{d} \tau-\frac{1}{2} \int_{0}^{t} J(\tau) P_{3}(\tau) \mathrm{d} \tau .
\end{aligned}
$$

With the known evolution operator, given by Eqs. (2) (3) and (8), the average spin polarization vector is obtained readily from the formal solution of the quantum Liouville equation

$$
\boldsymbol{P}(t) \cdot \boldsymbol{\sigma}=U(t)(\boldsymbol{P}(0) \cdot \boldsymbol{\sigma}) U^{\dagger}(t)=U(t) A(t),
$$

where part of the rhs is an auxiliary $2 \times 2$ matrix $A(t) \equiv$ $(\boldsymbol{P}(0) \cdot \boldsymbol{\sigma}) U^{\dagger}(t)$. For a general case of the initial spin polarization vector $\boldsymbol{P}(0)=\left(P_{1}(0), P_{2}(0), P_{3}(0)\right)$, one finds the matrix elements

$$
\begin{aligned}
& A_{11}(t)=P_{3}(0) U_{11}^{*}+\left[P_{1}(0)-\mathrm{i} P_{2}(0)\right]\left(-U_{21}\right), \\
& A_{12}(t)=P_{3}(0) U_{21}^{*}+\left[P_{1}(0)-\mathrm{i} P_{2}(0)\right] U_{11}, \\
& A_{21}(t)=\left[P_{1}(0)+\mathrm{i} P_{2}(0)\right] U_{11}^{*}-P_{3}(0)\left(-U_{21}\right), \\
& A_{22}(t)=\left[P_{1}(0)+\mathrm{i} P_{2}(0)\right] U_{21}^{*}-P_{3}(0) U_{11} .
\end{aligned}
$$

Since in Eq. (12) the rhs is a known $2 \times 2$ matrix, and because the lhs is the $2 \times 2$ matrix

$$
\boldsymbol{P}(t) \cdot \boldsymbol{\sigma}=\left[\begin{array}{cc}
P_{3}(t) & P_{1}(t)-\mathrm{i} P_{2}(t) \\
P_{1}(t)+\mathrm{i} P_{2}(t) & -P_{3}(t)
\end{array}\right],
$$

by equating the corresponding elements of the two matrices one determines the components of the spin polarization vector at a time $t$. One finds

$$
\begin{aligned}
& P_{1}(t)=\Re\left\{U_{11}(t) A_{12}(t)-U_{21}^{*} A_{22}(t)\right\}, \\
& P_{2}(t)=\Im\left\{U_{11}(t) A_{12}(t)-U_{21}^{*} A_{22}(t)\right\}, \\
& P_{3}(t)=\Re\left\{U_{11}(t) A_{11}(t)-U_{21}^{*} A_{21}(t)\right\} .
\end{aligned}
$$

Let us note that the magnitude of $\boldsymbol{P}(t)$ is a constant throughout the evolution and is, in fact, equal to one for a pure state.

\section{Calculation of phases}

Despite the existence of a number of analytical solutions to the time-dependent Schrödinger equation for relatively simple examples describing a driven two-level system provided in [12], these are mainly not translated to the corresponding analytical expressions for the phases and one has to turn to numerical analysis. This nevertheless enables an efficient analysis in a number of cases. We illustrate the typical results for the total, dynamic and geometric phases, obtained from Eqs. (10), (11) and (6). For a typical case considered in [12], namely the choice (Eq. (19) in [12])

$$
q(t)=\exp \left((-2 / a) \sinh ^{2}(\sqrt{a} h t / 2)\right),
$$

with a real constant $a \leq 2$, and the corresponding driving field is from Eq. (3)

$$
\frac{J(t)}{h}=\frac{\frac{1}{a} \sinh ^{2}(\sqrt{a} h t)-2 \sinh ^{2}(\sqrt{a} h t / 2)}{\sqrt{\mathrm{e}^{\frac{4}{a} \sinh ^{2}(\sqrt{a} h t / 2)}-\frac{1}{a} \sinh ^{2}(\sqrt{a} h t)-1}} .
$$

For such a driving field, the total, dynamic and geometric phases, as functions of time $t$ are plotted in Figs. 1 and 2 for $h=1$ and $a=\frac{2}{3}$.

It is seen that from an initial time $t=0$ to a final $t=10$, during which the driving field $J(t)$ is appreciably different from zero, the corresponding geometric phase changes (by $\Delta \beta \approx 0.8$ radians) thus enabling detection. Analogous results are also obtained for other driving fields considered in [12].

\section{Conclusions}

Phase is a fundamental notion in quantum mechanics, and in particular the study of geometric phases is an attempt to understand quantum mechanics better. It is known that the total phase and visibility are directly observable in an interference experiment [23]. Geometric phases have some implications in quantum information theory. These phases can form the basis of any quantum computation $[24,25]$, resilient to certain types of errors. It offers the potential of a fault-tolerant way of performing geometric quantum computation. Two references concerning the robustness of the geometric phase for non-Abelian gates and Berry phase are [26, 27].

Here we determine the geometric phase corresponding to a new type of driven two-state system [12] which is thus of relevance to qubit control. The main result of 


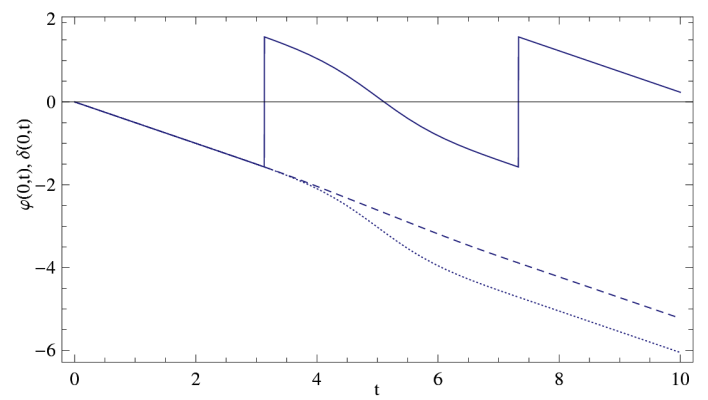

Fig. 1. Total phase $\varphi(0, t)$ (solid curve) and dynamic phase $\delta(0, t)$ (dashed curve), in radians, for driven time-dependent two-level quantum system, with the Hamiltonian (1), and in the case of the driving field Eq. (16). The initial spin polarization vector is $\boldsymbol{P}(0)=(1,0,0)$, completely along the $x$-axis, while the two other constants are $h=1$ and $a=\frac{2}{3}$. It is seen that the dynamic phase is a smooth, monotonically decreasing function of the elapsed time $t$. The two jumps by $+\pi$ radians in the total phase, stem from the multiple-valued arctan function appearing in Eq. (10) for the total phase, represent the change of sign in the probability amplitude, and are experimentally observable. By using the principal value of the function arctan, one removes the jumps and obtains for the total phase the dotted curve.

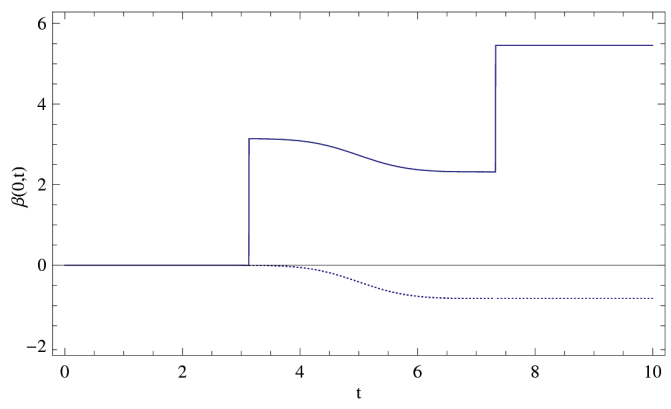

Fig. 2. Geometric phase $\beta(0, t)$, in radians (solid curve), for driven time-dependent two-level quantum system, with the Hamiltonian (1), and in the case of the driving field Eq. (16). It is seen that there is a considerable change in the geometric phase of $\approx 5.5$ radians (solid curve). By removing the jumps in the total phase (there are two jumps of $+\pi$ ), one finds a slight net decrease in geometric phase of $\approx 0.8 \mathrm{rad}$ (dotted curve). The initial spin polarization vector is $\boldsymbol{P}(0)=(1,0,0)$, while the two other constants are $h=1$ and $a=\frac{2}{3}$. The resulting geometric phase reflects the behavior of the total and dynamic phases presented in Fig. 1.

our work is contained in Eqs. (11) and (14), which give explicit expressions for computing geometric phase for a given evolution.

\section{Acknowledgments}

This work was supported by the Ministry of Science and Education of the Republic of Serbia, contracts No. 171006, No. 171017, No. 171020, No. 171028, and No. 171038, and by COST (Action MP1006).

\section{References}

[1] L. Landau, Phys. Z. Sov. 2, 46 (1932).

[2] C. Zener, Proc. R. Soc. A 137, 696 (1932).

[3] I. Rabi, Phys. Rev. 51, 652 (1937).

[4] E.T. Jaynes, F.W. Cummings, Proc. IEEE 51, 89 (1963).

[5] S.L. McCall, E.L. Hahn, Phys. Rev. 183, 457 (1969).

[6] S.E. Economou, L.J. Sham, Y. Wu, D.G. Steel, Phys. Rev. B 74, 205415 (2006).

[7] A. Greilich, S.E. Economou, S. Spatzek, D.R. Yakovlev, D. Reuter, A.D. Wieck, T.L. Reineckee, M. Bayer, Nature Phys. 5, 262 (2009).

[8] E. Poem, O. Kenneth, Y. Kodriano, Y. Benny, S. Khatsevich, J.E. Avron, D. Gershoni, Phys. Rev. Lett. 107, 087401 (2011).

[9] P.K. Jha, Y.V. Rostovtsev, Phys. Rev. A 81, 033827 (2010).

[10] A. Gangopadhyay, M. Dzero, V. Galitski, Phys. Rev. B 82, 024303 (2010).

[11] Q. Xie, W. Hai, Phys. Rev. A 82, 032117 (2010).

[12] E. Barnes, S. Das Sarma, Phys. Rev. Lett. 109 , 060401 (2012).

[13] M.V. Berry, Proc. R. Soc. Lond. A 392, 45 (1984).

[14] M.V. Berry, J. Phys. A, Math. Gen. 18, 15 (1985).

[15] Y. Aharonov, J. Anandan, Phys. Rev. Lett. 58, 1593 (1987).

[16] J. Samuel, R. Bhandari, Phys. Rev. Lett. 60, 2339 (1988).

[17] S. Pancharatnam, Proc. Indian Acad. Sci. A 44, 247 (1956).

[18] Collected Works of S. Pancharatnam, Oxford University Press, London 1975.

[19] D.M. Tong, J.L. Chen, J.F. Du, Chin. Phys. Lett. 20, 793 (2003)

[20] I. Mendaš, Phys. Rev. A 55, 1514 (1997).

[21] I. Mendaš, Phys. Rev. A 67, 044101 (2003).

[22] M.A. Bouchene, M. Abdel-Aty, Phys. Rev. A $\mathbf{7 9}$, 055402 (2009).

[23] E. Sjöqvist, A.K. Pati, A. Ekert, J.S. Anandan M. Ericsson, D.K.L. Oi, V. Vedral, Phys. Rev. Lett. 85, 2845 (2000).

[24] A. Ekert, M. Ericsson, P. Hayden, H. Inamori, J.A. Jones, D.K.L. Oi, V. Vedral, J. Mod. Opt. 47, $2501(2000)$

[25] J.A. Jones, V. Vedral, A. Ekert, G. Castagnoli, $N a-$ ture 403, 869 (2000).

[26] M. Johansson, E. Sjöqvist, I. Mauritz Andersson, M. Ericsson, B. Hessmo, K. Singh, D.M. Tong, Phys. Rev. A 86, 062322 (2012).

[27] S. Filipp, J. Klepp, Y. Hasegawa, C. Plonka-Spehr, U. Schmidt, P. Geltenbort, H. Rauch, Phys. Rev. Lett. 102, 030404 (2009). 